\title{
"Challenges of Sign Language Interpreters in their work with seriously ill and dying deaf people." A qualitative investigation.
}

Ruszynski, Anuschka M. ${ }^{1}$, Orellana-Rios, Claudia L. ${ }^{2}$, Becker, Gerhild ${ }^{2}$

1 Protestant Deaconess House Berlin, Germany

${ }^{2}$ Clinic for Palliative Care, Medical Centre, University of Freiburg, Faculty of Medicine, Germany

Contact: anuschka.maria.ruszynski@posteo.de

\section{Background}

Since 2002, German Sign Language has been legally recognized as an independent language. Deaf people are entitled by Social Insurance Code I,§17, Abs.2, to use a Sign Language Interpreter (SLI) in settings of social benefits or medical treatment Therefore, SLIs play a vital role in deaf people's lives, because they enable communication with hearing people.

Communication is regarded as the second pillar in palliative medicine (Husebø, 2009) and "is an indispensable part of therapy - sometimes it is the only possibility of therapy in palliative medicine" (Aulbert 2012, p. 1037)

In deaf people's case, communication takes place under "six eyes": doctor, patient and SLI. The SLI must maintain direct visual contact to the patient at all times and must translate every word respectively every sign, facial expression and posture as an essential part of sign language.

However, SLIs are neither experts for deaf people nor experts for medicine. They are rather experts for communication. Up to this point no study has investigated the experiences of SLIs in the palliative care context.

\section{Aim}

The aim of the qualitative study was to explore the challenges SLIs are

confronted with while interpreting for seriously ill and dying deaf people and how do they deal with these challenges.

\section{Methods}

- Semi-structured interviews were carried out with six SLIs.

- Questions were asked about the translation process, the impact of the topic death and dying on the professional role, and the impact of this topic on the SLIs' personal life.

- The interview partners were recruited via the Federal Association of Sign Language Interpreters Germany e.V.

- The interviews were audio taped, transcribed verbatim and analyzed with the content analysis method (Mayring, 2015).

\section{Results}

The challenges of the SLIs can be summarized in 9 main categories:

1. Experiences with deaf people

The interviewees reported to be either children of deaf parents, or to have had experience interpreting for seriously ill deaf people

2. Acquisition of knowledge for interpreting in the medical field

Interviewees acquired medical knowledge from their education, from previous occupations, from conversations with family members

or simply from practice.

3. Interpreting for a seriously ill and dying deaf: personal and professional challenges

Interviewees described personal and professional challenges, e.g. responsibility, emotional involvement, remain

professional in accordance with the code of conduct for SLIs (confidentiality, neutrality)

4. Sign language communication in palliative situations

There were several reported challenges including dealing with the deteriorating health of patients, physical changes that greatly impact the ability to sign, simple things like narrow hospital rooms (sign language needs space), the topic of death and dying itself and SLIs strategies to deal with it. 5. Specifics of Deaf culture

Communication requires more tools (e.g. illustrations through pictures) than for hearing people. Deaf people, however, cannot look at a picture and watch the gestures of the translated at the same time. Therefore, more time is needed. SLIs felt responsible for explaining the peculiarities of both sides to those involved: deaf people \& health care professionals.

6. Building and shaping relationships with patient, doctor, nurse, family

Interviewees named sympathy as the basis for a trusting cooperation with the deaf patient.

7. Perception of the situation of serious ill and dying deaf people

SLI's talk about the desires and needs of deaf patients and the relationships they had with their doctors and family members

8. Personal strategies and resources

Strategies mentioned were e.g.supervision, awareness, trust and sympathy, saying goodbye and life-experience.

9. "Good interpreting" for a seriously ill and dying deaf patient

Reflection of the own narration and opinion: Good interpreting needs e.g. more time, intensive

cooperation with health care professionals, more knowledge about palliative care

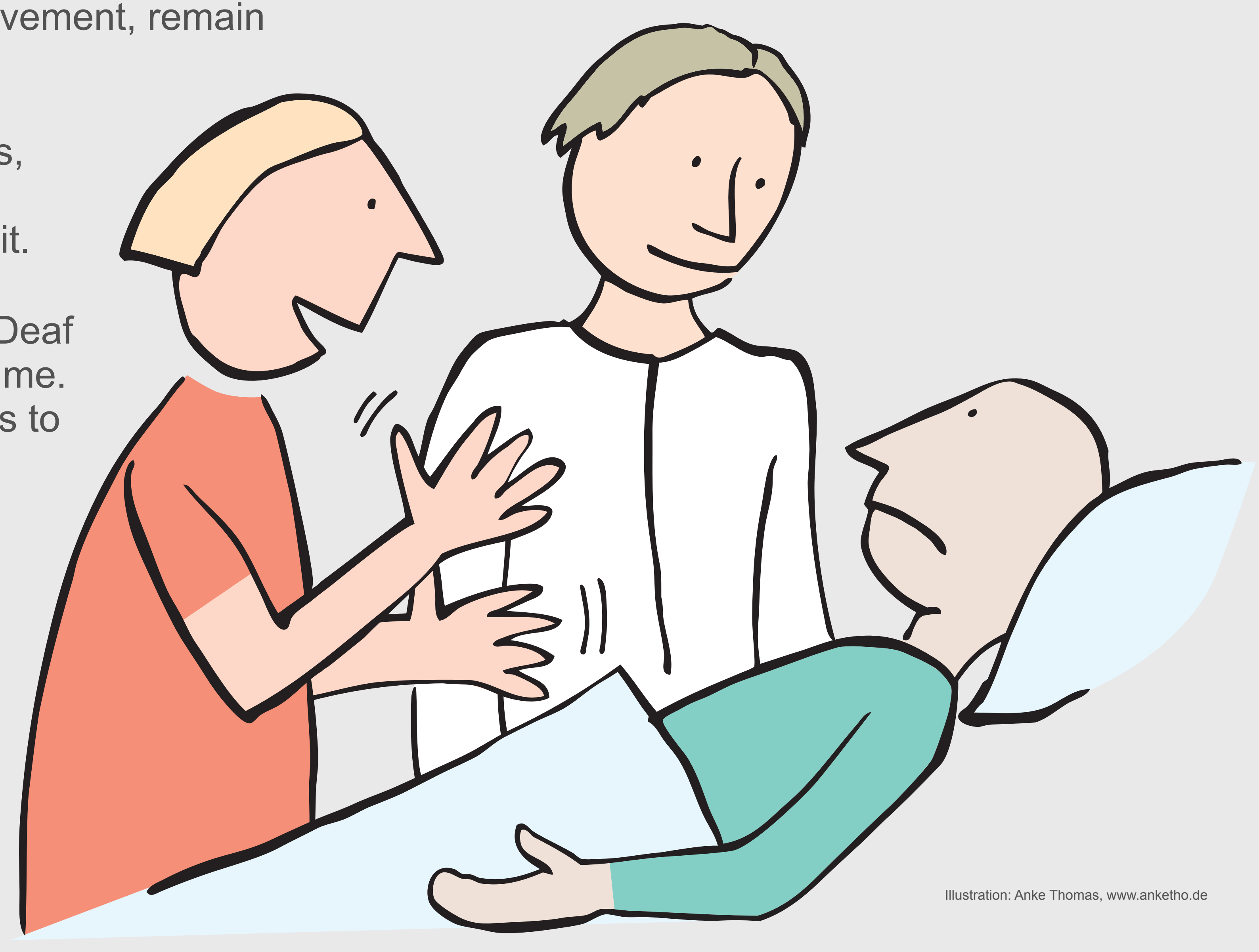

"Die Sachen müssen einmal durch uns durch und durch die Hände wieder raus."

\section{Conclusions}

- Sign language interpretation in palliative care needs to be deemed a highly complex job with personal and professional challenges

- At the same time, the interviews showed that a palliative situation was manageable for SLIs through an individually reflected way of working and behaving.

- SLls felt responsible for the quality of palliative care.

- SLIs are faced with various challenges in palliative care that they may not have otherwise been prepared for in professional training. Subject-specific courses in palliative care could be beneficial for SLIs. 